\title{
Editing HIV entry
}

A genome-editing approach targeting the CC-chemokine receptor CCR5, which is the main co-receptor for entry of HIV-1 into CD4 ${ }^{+} \mathrm{T}$ cells, has the potential to reconstitute immune function in $\mathrm{HIV}^{+}$individuals by creating an HIV-resistant CD4 ${ }^{+}$ T-cell population, according to a recent report in Nature Biotechnology.

Individuals who are homozygous for the $\triangle 32$ deletion in CCR 5 are resistant to HIV-1 infection, which makes CCR5 a potential target for HIV therapy. In this study, Perez et al. sought to permanently disrupt the endogenous CCR5 gene in $\mathrm{CD} 4^{+}$ $\mathrm{T}$ cells - to mimic the $\triangle 32 C C R 5$-null genotype - using zinc-finger nucleases (ZFNs). The engineered ZFNs create double-strand breaks at specific sites in the genome, as determined by the sequence specificity of the DNA-binding domain, that are then imperfectly repaired and permanently disrupt the genomic sequence.

The authors designed ZFNs that target the DNA sequence encoding the first transmembrane domain of CCR5 and showed that in a reporter cell line expressing CCR5, transduction with the CCR5-targeted ZFNs
(CCR5-ZFNs) resulted in high efficiency (50-80\%) target-gene mutation and a more than tenfold decrease in cell-surface expression of CCR5. By analysing the consensus sequence of the ZFN-binding site in CCR5, 15 putative alternative cleavage sites were identified throughout the genome. In support of the specificity of the engineered ZFNs, there was no detectable ZFN activity at any of these sites with the exception of a low level of modification at CCR2, which is not predicted to result in a marked phenotype, and a very low frequency modification of ABLIM2, a gene that is not expressed in T cells and that is also not predicted to have adverse effects.

In vitro, cells transduced with the CCR5-ZFNs had decreased levels of infection with a CCR5-tropic HIV-1 isolate than non-transduced cells or cells transduced with a control ZFN. When a $\mathrm{CD} 4^{+} \mathrm{T}$-cell line was transduced with suboptimal levels of CCR5-ZFNs (to create a low baseline level of endogenous CCR5 disruption), infection with HIV-1 resulted in a 30 -fold increase in the percentage of $\mathrm{T}$ cells with

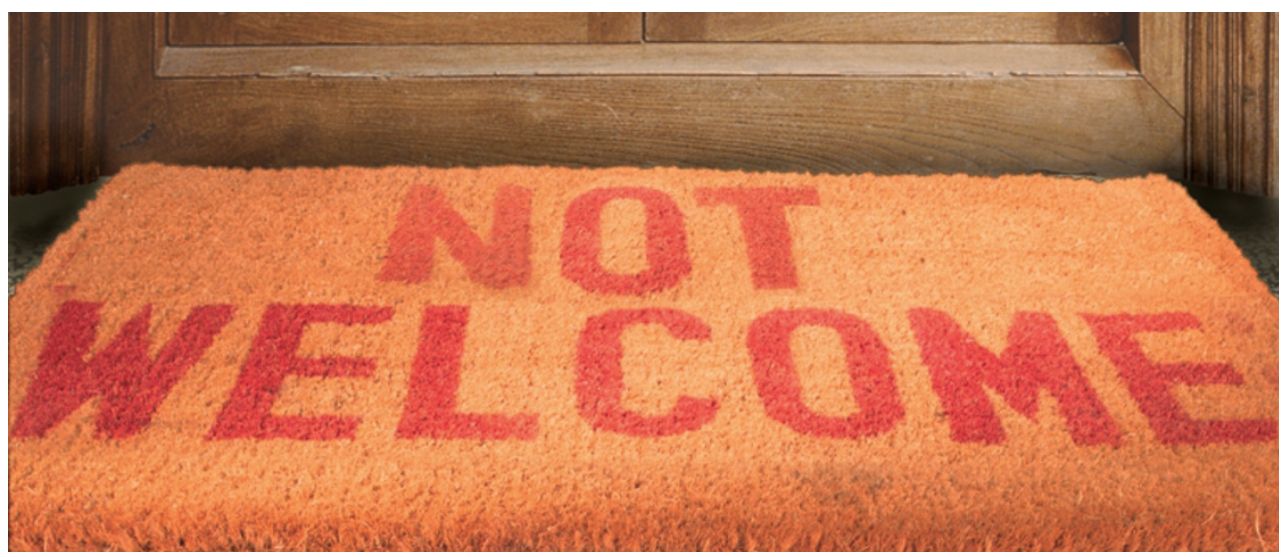

ZFN-modified CCR5 alleles after 52 days of culture. Therefore, ZFN-mediated disruption of CCR5 confers a long-term survival advantage on $\mathrm{CD}^{+} \mathrm{T}$ cells in the presence of HIV-1 infection. This in vitro survival advantage against HIV-1 infection that was provided by the transduction with CCR5-ZFNs was confirmed for primary human $\mathrm{CD} 4^{+}$ $\mathrm{T}$ cells isolated from healthy donors expressing wild-type CCR5.

To explore the clinical feasibility of this approach, primary human $\mathrm{CD} 4^{+} \mathrm{T}$ cells were transduced with CCR5-ZFNs and propagated in culture before adoptive transfer to severely immunodeficient mice. After one month of HIV-1 infection, there was a threefold enrichment for ZFN-disrupted CCR5 alleles in HIV-1-infected mice compared with non-infected mice, which indicates that CCR5 disruption also confers a survival advantage on $\mathrm{CD} 4^{+} \mathrm{T}$ cells in vivo. Mice engrafted with CCR5disrupted $\mathrm{CD} 4^{+} \mathrm{T}$ cells compared with control $\mathrm{CD} 4^{+} \mathrm{T}$ cells had markedly decreased plasma viraemia after HIV-1 infection, which shows that the modified cells confer resistance to HIV-1 infection.

The authors conclude that these results "support the clinical development of adoptive immunotherapy to reconstitute the memory cell pool of HIV-infected patients with ZFNmodified CD4 ${ }^{+} \mathrm{T}$ cells", although the approach must first be analysed in terms of chronic infection of non-human primates.

Kirsty Minton

ORIGINAL RESEARCH PAPER Perez, E. E. et al. Establishment of HIV-1 resistance in $\mathrm{CD} 4^{+} \mathrm{T}$ cells by genome editing using zinc-finger nucleases. Nature Biotech. 26, 808-816 (2008) 